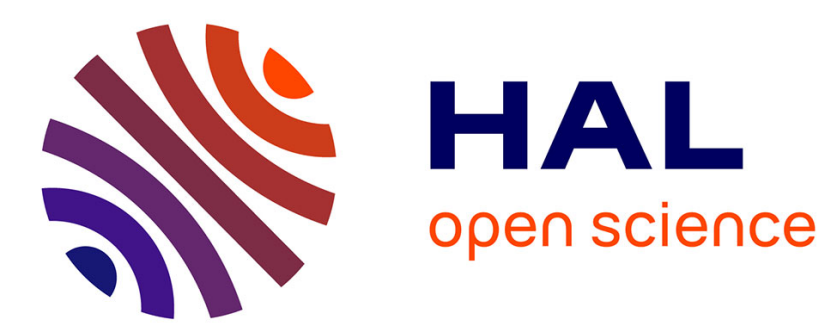

\title{
RELAXATION OF 3d AND 4f ELECTRONS IN METALS AND ALLOYS
}

\author{
L. Hirst
}

\section{To cite this version:}

L. Hirst. RELAXATION OF 3d AND 4f ELECTRONS IN METALS AND ALLOYS. Journal de Physique Colloques, 1974, 35 (C6), pp.C6-21-C6-31. 10.1051/jphyscol:1974603 . jpa-00215703

\section{HAL Id: jpa-00215703 https://hal.science/jpa-00215703}

Submitted on 1 Jan 1974

HAL is a multi-disciplinary open access archive for the deposit and dissemination of scientific research documents, whether they are published or not. The documents may come from teaching and research institutions in France or abroad, or from public or private research centers.
L'archive ouverte pluridisciplinaire HAL, est destinée au dépôt et à la diffusion de documents scientifiques de niveau recherche, publiés ou non, émanant des établissements d'enseignement et de recherche français ou étrangers, des laboratoires publics ou privés. 


\title{
RELAXATION OF 3d AND 4f ELECTRONS IN METALS AND ALLOYS
}

\author{
L. L. HIRST \\ Institut für Theoretische Physik der Universität Frankfurt \\ 6 Frankfurt a M, German Federal Republic
}

\begin{abstract}
Résumé. - On présente une revue des études de relaxation électronique d'impuretés $3 d$ et $4 \mathrm{f}$ dans les métaux effectués à l'aide des spectres Mössbauer de relaxation lente. Nous soulignons particulièrement l'effet d'éclatements de type ionique - comme les éclatements d'impuretés $4 \mathrm{f}$ par l'effet du champ cristallin - et les effets de réarrangement interconfigurationnel lent dans les spectres Mössbauer đ'émission. Les informations obtenues par spectroscopie Mössbauer sont comparées à celles déduites par RPE.
\end{abstract}

\begin{abstract}
The study of the electronic relaxation of $3 \mathrm{~d}$ and $4 \mathrm{f}$ impurities in metals by means of their slow-relaxation Mössbauer spectra is reviewed. We emphasize especially the effect of ionictype splittings, such as crystal-field splittings of $4 \mathrm{f}$ impurities ; and the effects of slow intraconfigurational rearrangement in Mössbauer source spectra. The information obtainable from the Mössbauer method is compared with that obtainable from EPR.
\end{abstract}

1. Introduction : relaxation in the Mössbauer effect. - The study of slowly-relaxing dilute paramagnetic impurities in metals is an interesting application of the Mössbauer effect which has only recently been developed [1-14]. In this talk, we shall seek to summarize the results obtained so far and to point to interesting problems awaiting future investigation.

Let us begin by reviewing briefly the basic features of relaxation in the Mössbauer effect. (Although we shall be interested in the relaxation of an impurity due to the conduction electrons of a metal, these basic features are similar for relaxation by any mechanism. Studies of relaxation in insulators $[15]$ and in concentrated intermetallic compounds [16] predated work on dilute impurities in metals.)

To fix ideas, let us first consider a $4 f$ impurity, supposing that only its ground level in the crystalline electric field (CEF) is important. The electronic degrees of freedom within this CEF ground level are symbolized by a spin $S^{\prime}$ (actually a fictitious spin). The nuclear spin, in either the internal ground state or the Mössbauer excited state of the nucleus, is denoted $I$. The system which drives the relaxation (the conduction electrons in our case) is called the bath; it is assumed to remain always in thermal equilibrium. There is a hyperfine coupling $H_{\mathrm{hf}}$ between $I$ and $S^{\prime}$. Also there is a coupling between $S^{\prime}$ and the bath (called the k-f coupling in the case of $4 \mathrm{f}$ impurities and conduction electrons) which is responsible for the relaxation. In the simplest cases, the relaxation of $S^{\prime}$ induced by the $k-f$ coupling can be characterized by an electronic relaxation rate $1 / t_{\mathrm{el}}$, which depends upon the strength of the $\mathrm{k}-\mathrm{f}$ coupling and the temperature of the bath. To qualitatively discuss the influence of relaxation on the Mössbauer spectrum, we compare $1 / t_{\mathrm{el}}$ with two other frequencies; $1 / t_{\mathrm{nuc}}$, the lifetime broadening corresponding to the finite lifetime of the internal excited state of the nucleus against gamma emission; and $\omega_{\mathrm{hf}}$, the total hyperfine splitting in frequency units. Normally $1 / t_{\text {nuc }}$ is small compared to $\omega_{\mathrm{hf}}$; in fact this must be the case if relaxation phenomena are to be conveniently observable.

In figure 1 we indicate schematically a typical behaviour of the Mössbauer spectrum as the electronic relaxation rate, $1 / t_{\mathrm{e}}$, increases from very slow to very fast. In the limit of very slow relaxation, the bath has no effect and we see a spectrum corresponding simply to the hyperfine splitting of the ion in the paramagnetic

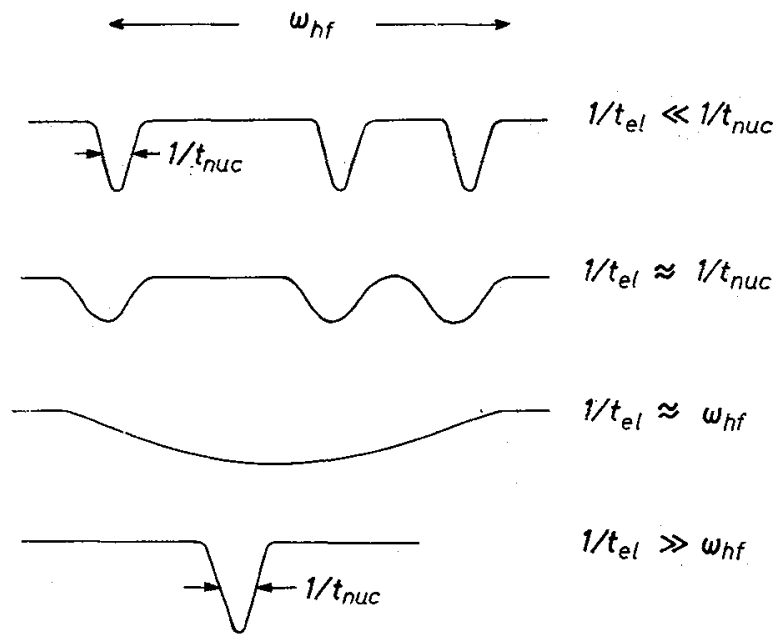

FIG. 1. - Schematic illustration of the form of the Mössbauer spectrum as the electronic relaxation rate, $1 / t_{\mathrm{el}}$, ranges from slow to fast, for the simplest case of a single electronic level. The total area under all curves should be equal, but the vertical scale has been varied for graphical clarity. The horizontal axis is the Doppler velocity in frequency units $(v / c) E_{y} / \hbar$ 
state, with nuclear-lifetime broadening. As $1 / t_{\mathrm{e} 1}$ becomes comparable to $1 / t_{\text {nuc }}$, the lines of the zerorelaxation spectrum broaden, and as $1 / t_{\mathrm{e} 1}$ becomes comparable to $\omega_{\mathrm{hf}}$ they fuse into a single broad peak. Finally, as $1 / t_{\mathrm{e} 1}$ becomes large compared to $\omega_{\mathrm{hf}}$ the broad peak collapses into a single narrow line of width $1 / t_{\text {nuc }}$. (This describes the intrinsic spectrum of the absorber or source containing the relaxation behaviour of interest. The experimental spectrum will be folded with a single-line source or absorber resonance, giving an extra broadening of order $1 / t_{\text {nuc }}$.)

The behaviour just discussed was for the case without an external magnetic field or magnetic ordering. It should be emphasized that in the slow-relaxation regime one obtains a spectrum with resolved magnetic hyperfine splitting even without a magnetic field. The basic relaxation information can generally be obtained most conveniently from such zero-field spectra, although spectra in finite fields may yield useful supplementary information [13]. The collapse into a narrow-line spectrum in the fast-relaxation limit corresponds to the nucleus seeing a well-defined time average of the rapidly-fluctuating hyperfine fields generated by the electronic moment. In the case without an external field or magnetic ordering this time-averaged hyperfine field contains no magnetic part; but in cases with lower-than-cubic symmetry it will in general contain an electric quadrupole part. Then in the fast-relaxation limit there will be a hyperfine splitting of quadrupolar type, which however is typically smaller than a magnetic splitting and may not be well resolved.

The collapse to a line or lines of width $1 / t_{\text {nuc }}$ in the fast-relaxation limit is a consequence of the assumption that the relaxation forces act exclusively on the electronic spin $S^{\prime}$. To the extent that there were relaxation forces acting directly on the nuclear spin $I$, this collapse phenomenon would not occur. But the relaxation forces acting directly on the nuclear spin from an extra-ionic source are generally too small to have an appreciable effect on Mössbauer spectra, even though their effects are readily detectable in NMR.

It should also be remarked that the Mössbauer resonance is completely localized to a single ion, as a result of the fact that there exists no mechanism which could transfer the internal nuclear excitation from one ion to its neighbour with any significant probability. For this reason such phenomena as bottlenecking or exchange narrowing, which occur in EPR and amount basically to a delocalization of the resonance by transfer of the excitation energy from a given ion to its environment $[19,26]$, cannot occur in the Mössbauer effect.

The above discussion indicates that the range of $1 / t_{\mathrm{e} 1}$ values which sensitively affect the spectrum, and thus can be studied with the Mössbauer effect, extends from a fraction of $1 / t_{\mathrm{puc}}$ to several times $\omega_{\mathrm{hf}}$. In principle, one can study relaxation rates falling anywhere in this range. In practice, however, it is desirable to study a given impurity over a wide range of relaxation rates, by varying the temperature. It is particularly desirable to obtain spectra where the relaxation is sufficiently slow to leave a well-resolved hyperfine splitting. In the first place, the information about the electronic states of the impurity which can be obtained from the analysis of such slow-relaxation spectra may be needed in order to properly formulate the relaxation theory. Furthermore, even if the electronic states are known, it is desirable to see the slow-relaxation spectrum in order to be sure that the supposed relaxation broadening is not actually due to some other effect, such as incipient magnetic ordering in clusters of impurities.

,For $4 \mathrm{f}$ impurities in metals, it is usually, although not always, possible to obtain spectra exhibiting resolved splittings of the zero-field, slow-relaxation type at liquid-helium temperatures. For $3 \mathrm{~d}$ impurities, on the other hand, this has proved to be very difficult [17]. In the first place, the characteristic Mössbauer frequencies $1 / t_{\text {nuc }}$ and $\omega_{\mathrm{hf}}$ are an order of magnitude smaller for the main Mössbauer isotope, ${ }^{57} \mathrm{Fe}$, as compared to such $4 \mathrm{f}$ isotopes as ${ }^{166} \mathrm{Er}$ or ${ }^{170} \mathrm{Yb}$. (These rates are $10^{7}$ and $0.5 \times 10^{9} \mathrm{~s}^{-1}$ for ${ }^{57} \mathrm{Fe}$, as compared to $0.5 \times 10^{9}$ and $10^{10} \mathrm{~s}^{-1}$ for ${ }^{166} \mathrm{Er}$ or ${ }^{170} \mathrm{Yb}$.) In addition, the electronic relaxation is faster for $3 \mathrm{~d}$ as compared to $4 \mathrm{f}$ impurities due to the stronger coupling to the conduction electrons. As we have noted, the bottleneck effect, which renders the fast relaxation rates ineffective for the EPR of certain 3d impurities, does not apply to Mössbauer measurements. By cooling to very: low temperatures it may be possible to make the relaxation sufficiently slow (although nothing is gained by cooling below the Kondo temperature if there is one). In fact, by use of a dilution refrigerator spectra have recently been obtained for Fe-Pt which exhibit incipient zero-field splitting apparently of slow-relaxation origin [18]. In the present talk we will put the primary emphasis on $4 \mathrm{f}$ impurities, where the experimental situation is clearer. However, the basic principles involved are very similar in the two cases, and we hope that more detailed analyses of $3 \mathrm{~d}$ impurities can be made in the near future.

2. Information from the slow-relaxation limit. For $4 \mathrm{f}$ impurities in metals, one can often obtain lowtemperature Mössbauer spectra in which the relaxation rate is negligible, or at least small enough to enable one to estimate the positions and intensities of the lines in the slow-relaxation limit. From analysis of such spectra, one may be able to identify the impurity's CEF ground state, to fit its $g$-tensor and other properties, and to fit the hyperfine coupling constants [2]. The details of this analysis depend upon the system at hand, its symmetry, and the amount of independent information one may have available from other experiments.

One particularly interesting fact to emerge from the slow-relaxation spectra is the presence of an enhance- 
ment in the value of the magnetic hyperfine coupling constant, $A$. Whereas the value of $A$ is found to be the same to within $2 \%$ for a given ion of given valency in various insulator hosts [20], the value of $A$ in metals is found to be of the order of $10 \%$ larger. Similar enhancements were later also found by EPR, with accurate agreement where the same alloys were studied [23]. Some measured values are listed in Table I. It appears possible to understand this enhancement in terms of a local polarization of the conduction electrons by the electronic spin, $S$, via the exchange coupling. The polarized conduction electrons then act back on the nuclear spin, $I$, via the contact hyperfine interaction, yielding an effective extra coupling between $I$ and $S[21,22]$. Table $I$ indicates a general tendency for the enhancement of $A$ to increase with the density of conduction-electron states, as would be expected on the basis of this mechanism.

\section{TABLE I}

Values of the magnetic hyperfine coupling constant, $A$, for ${ }^{166} \mathrm{Er}^{3+}$ in various hosts as determined from slow-relaxation Mössbauer spectra. The constant $A$, in angular-frequency units, is defined by $H_{\mathrm{mhF}}=\hbar A \mathbf{I} . \mathbf{J}$, where $\mathbf{J}$ is the total electronic angular momentum. $A_{0}=1.52 \times 10^{9} \mathrm{~s}^{-1}$ denotes the average value of $A$ for insulator hosts [20].

$\begin{array}{llrl}\text { Host } & A\left(\mathrm{~s}^{-1}\right) & \left(A-A_{0}\right) / A_{0} & \text { Ref. } \\ \overline{\mathrm{Ag}} & 1.58 \times 10^{9} & \overline{4} \% & {[11]} \\ \mathrm{Au} & 1.58 \times 10^{9} & 4 \% & {[10]} \\ \mathrm{Th} & 1.62 \times 10^{9} & 7 \% & {[12]} \\ \mathrm{Y} & 1.65 \times 10^{9} & 9 \% & {[24]} \\ \mathrm{Zr} & \geqslant 1.74 \times 10^{9} & \geqslant 15 \% & {[2]}\end{array}$

As noted above, information from the slow-relaxation limit may be particularly important for setting up a quantitative theory for the finite-relaxation case.

3. The spectral density of a fermion bath. - We come now to our main theme, the relaxation caused by the conduction electrons. We want to examine the following questions : What relaxation is induced in the electronic shell of the impurity by the conduction electrons ? How does this electronic relaxation affect the Mössbauer spectrum ? How much information about the electronic relaxation, and hence about the coupling between impurity and conduction electrons, can be gained from Mössbauer spectroscopy, and how does this information compare with that which can be gained from other experimental methods such as EPR ?

For purposes of discussion it is convenient to separate two aspects of the relaxation caused by the conduction electrons. One aspect, which will be discussed in later sections, is the dependence of the relaxation on the form of the $\mathrm{k}-\mathrm{f}$ coupling. The other aspect, which we discuss now, is the dependence of the relaxation on frequency and temperature. This dependence is carried by a spectral density function [3, 25-28] $J_{0}(\omega, T)$, which describes the power spectrum exerted on the electronic shell of the impurity by the conduction-electron bath at temperature $T$. The relevant frequency depends upon the energy levels of the impurity. If we consider two impurity states separated by energy $\Delta$, then the rate of transition from the lower to the upper level induced by the bath is proportional to $J_{0}(\Delta, T)$, while the rate from the upper to the lower level is proportional to $J_{0}(-\Delta, T)$.

Assuming the conduction electrons to be noninteracting, it can be shown that they yield a spectral density $[25,26]$

$J_{0}(\omega, T)=2 \pi\left|I_{0} \rho\left(\varepsilon_{\mathrm{F}}\right)\right|^{2}\{\omega /[\exp (\omega / k T)-1]\}$.

Here the form of dependence on $\omega$ and $T$ results solely from the fermi statistics of non-interacting conduction electrons; it is obtained basically by folding the fermi distributions of the initial (occupied) and final (unoccupied) conduction-electron states. In particular, the form will be the same whether or not Bloch band structure of the conduction electrons is taken into account. In eq. (1), $\rho\left(\varepsilon_{\mathrm{F}}\right)$ is the density of conduction-electron states per spin direction per unit-cell volume, and $I_{0}$ characterizes the strength of the $\mathrm{k}-\mathrm{f}$ coupling. For a $4 \mathrm{f}$ impurity in a metal, the value of $\left|I_{0} \rho\left(\varepsilon_{\mathrm{F}}\right)\right|^{2}$ is typically $10^{-4}$ to $10^{-3}$. The \{\} bracket in eq. (1) simplifies in limiting cases as follows :

$$
\begin{aligned}
& |\omega| \ll k T:\{\} \approx k T \\
& \omega \gg k T:\{\} \approx \omega \exp (-\omega / k T) \\
& -\omega \gg k T:\{\} \approx|\omega| \text {. }
\end{aligned}
$$

We shall be interested in the frequency dependence of the spectral density mainly in the context of a $4 f$ impurity subject to CEF splitting. Eq. (2a) shows that the transition rates between different electronic states within a given CEF level are proportional to $T$, which is the familiar Korringa law. However, if we consider transitions between states belonging to different CEF levels, we obtain large deviations from the Korringa law when the separation $\Delta$ of the two levels is made larger than $T$. The transition rate from the lower to the higher level is decreased, while that from the higher to the lower level is increased and remains finite even at $T=0$. The rate of transition from the higher to the lower level is faster than that from the lower to the higher according to the relation

$$
J_{0}(-\omega, T)=\exp (\omega / k T) J_{0}(\omega, T) .
$$

This may be called the equilibration relation, since it guarantees that an arbitrary initial population of the impurity states will be brought toward a thermal population distribution at the bath temperature by the relaxation forces. It can be shown to hold quite generally for any type of thermal bath [28].

In summary, the single electronic relaxation rate 
$1 / t_{\mathrm{e} 1}$ contemplated in our introductory discussion has to be replaced by a spectral density function in order to obtain a realistic relaxation theory for an impurity subject to ionic-type splittings. The form of the spectral density is determined by the conduction-electron bath, but the frequencies sampled depend upon the splittings of the particular impurity at hand.

4. Absorber and source spectra with impurity splitting. - In the last section, we discussed the electronic relaxation when the impurity is subject to ionic-type splittings. In the present section, we consider the form of the Mössbauer spectrum when such splittings are taken into account. Although the same basic discussion would apply in principle to any type of splitting of $3 \mathrm{~d}$ or $4 \mathrm{f}$ impurities, the splittings of main interest up to now have been the CEF splittings of a $4 f$ impurity.

For concreteness, let us therefore consider a $4 f$ impurity with two CEF levels separated by energy $\Delta$. Since the gamma transition is caused by a purely nuclear operator, it will leave the electronic shell of the impurity in the same CEF level (assuming a CEF splitting much larger than the hyperfine interaction, as is normally the case). Therefore, if we first suppose our impurity to have negligible coupling to the bath, the Mössbauer absorber spectrum will be a superposition of two independent spectra, one for each of the CEF levels. The relative intensity of the two contributions is determined by the thermal populations of the two levels. Let us first assume $T \ll \Delta$; then the absorber spectrum is practically identical with that corresponding to the CEF ground level.

When we now consider a finite $k-f$ coupling which produces finite relaxation rates, the intrinsic spectrum of each CEF level may show relaxation effects, similar to those discussed for a single level in the introduction. If we had no relaxation transitions between the two levels, then in general we would expect the electronic relaxation within each of the two levels to be similar, and to increase in proportion to the temperature. However, as discussed previously, relaxation transitions between the two CEF levels do occur; and when $T \ll \Delta$ the rate of relaxation transitions from the higher to the lower level will be large compared to the transitions within either level. We emphasize again that this transition rate from the higher to the lower level remains large even at arbitrarily low temperatures. Therefore, the intrinsic spectrum of an excited CEF level will usually correspond to intermediate or fast relaxation even at the lowest temperatures. Inter-level relaxation transitions from the lower to the upper level will also occur and will cause the intrinsic spectrum of the lower level to show increased relaxation effects; however, the contribution due to such processes is typically small compared to the intra-level Korringa contribution unless $T \gtrsim \Delta$.

Our discussion indicates that the excited CEF level may have an intrinsic Mössbauer spectrum containing interesting relaxation effects even at the lowest tem- peratures ; however, there is no way for us to study this spectrum in a conventional absorber experiment, since at low temperatures the thermal population of the excited CEF level will be negligible. As we shall now discuss, the contributions from such a level may show up in the Mössbauer source spectrum due to slow rearrangement following the $\beta$-decay (or other nuclear transformation) [14]. It is most convenient to discuss these effects by comparing the spectra to be expected for the same impurity as absorber and source. However, it should be emphasized that the alloys used in such experiments must be extremely dilute in order to avoid undesired side effects from interactions between the impurities. For this reason, up to the present all spectra have been measured in the source, and an experimental source-absorber comparison would be quite impractical in most cases.

In a source experiment, the emission of the Mössbauer gamma quantum is prepared by a $\beta$-decay or other nuclear transformation, which (for almost all Mössbauer isotopes) changes the nuclear charge. This sudden change in the nuclear charge causes an extremely violent disruption of the electronic shell of the impurity, which cascades through many excited states before reaching the electronic ground state corresponding to the new nuclear charge (or more precisely, a thermal distribution of such states). We refer to this as the electronic rearrangement. The Mössbauer source spectrum will not be influenced by those rearrangement processes which are completed within a time much shorter than the nuclear lifetime of the Mössbauer excited state, $t_{\text {nuc }}$. For the $4 \mathrm{f}$ isotopes ${ }^{166} \mathrm{Er}$ and ${ }^{170} \mathrm{Yb}$, we have $t_{\text {nuc }} \approx 2 \times 10^{-9} \mathrm{~s}$, and we might take $10^{-10} \mathrm{~s}$ as a suitable line of demarcation defining extreme fast rearrangement. (In ref. [14] we erred in defining extreme fast rearrangement by comparison to $\omega_{\mathrm{hf}}$, but the numerical value given was correct.) Semi-quantitative estimates [14] show that the high-energy parts of the rearrangement, such as the rearrangement bringing the impurity into its stable configuration $4 \mathrm{f}^{n}$ for the new nuclear charge, will proceed very fast compared to $10^{-10} \mathrm{~s}$. This configurational rearrangement will leave a random population of the various intraconfigurational levels within the ground configuration, including the excited $[S L]-J$ manifolds. Estimates show that the highenergy part of the intraconfigurational rearrangement, bringing the impurity into its $[S L]-J$ ground manifold, is also fast compared to $10^{-10} \mathrm{~s}$. This leaves a random population of the CEF levels within this manifold. The rearrangement among CEF levels, especially the low-energy ones, is not necessarily faster than $10^{-10} \mathrm{~s}$; after all, rearrangement is relaxation and we know that relaxation among CEF levels may be slow on the Mössbauer time scale. Let us denote as $w_{\mathrm{e} \rightarrow \mathrm{g}}$ the rate of transition from an excited CEF level to the ground level, which may be assumed to result predominantly from conduction-electron processes. From eq. (2c) we see that $w_{\mathrm{e} \rightarrow \mathrm{g}}$ tends to be faster the higher the energy 
of the CEF level in question. When $w_{\mathrm{e} \rightarrow \mathrm{g}}>1 / t_{\text {nuc }}$, the tendency of the excited CEF level to depopulate by relaxation processes before the nuclear radiation is completed causes the intensity contributed by the excited CEF level to be smaller than that from the CEF ground level by a factor of about $\left(t_{\text {nuc }} w_{\mathrm{e} \rightarrow \mathrm{g}}\right)^{-1}$. If the excited level is more than a few $K$ above the ground level, then its contribution will usually be smaller than that from the ground level; but levels as high as 20 or $30 \mathrm{~K}$ will typically continue to contribute appreciably. Since the initial population of the excited CEF levels in a source does not depend upon the temperature, and since $w_{\mathrm{e} \rightarrow \mathrm{g}}$ approaches a finite low-temperature limit, the contributions from the excited CEF levels in the Mössbauer source spectrum remain finite as the temperature goes to zero. This should be contrasted with the absorber spectrum, in which the excited-level contributions approach zero at zero temperature. The difference between the source and absorber spectra disappears as the temperature is made large compared to the relevant CEF energies.

Once the basic physics has been recognized, the difference between the source and absorber cases turns out to be quite trivial from the point of view of the formal theory of relaxed Mössbauer spectra. It is merely necessary to substitute a fully random population distribution over all the relevant electronic states (i. e. a distribution in which each state has equal population probability) in place of the thermal population distribution appearing in the absorber theory [3]. In this way, the slower rearrangement processes are taken care of completely automatically. From a practical point of view, the computations do become more complicated because it may be necessary to take several CEF levels into account. Without going into the details of the quantitative theory, we show in figure 2 a model computation for a realistic $4 f$ system exhibiting the effects which we have just discussed [14]. The model has two nearly-degenerate CEF excited levels at about $20 \mathrm{~K}$, which contribute a broad central intensity but no recognizable peaks to the source spectrum. In other cases, contributions from the higher CEF levels might produce a weak central peak.

We have been discussing the effects of a random, non-thermal electronic initial population, produced by the $\beta$-decay, on the source spectrum. As has been pointed out by Hartmann, Gonzalez, and Imbert [7, 8], one may also have effects resulting from the nonthermal initial population of the nuclear spin levels, or more precisely of the nucleonic hyperfine levels within the CEF ground level. These authors were considering ${ }^{170} \mathrm{Yb}: \underline{\mathrm{Au}}$, for which the effects of slow electronic rearrangement are negligible, due to the absence of low-lying CEF levels and an unusually strong $\mathrm{k}-\mathrm{f}$ coupling. Then the rearrangement into the CEF ground level may be regarded as fast, and produces a random population of the hyperfine states within this level. This means that in the extreme slow relaxation limit the various hyperfine lines of the spec-

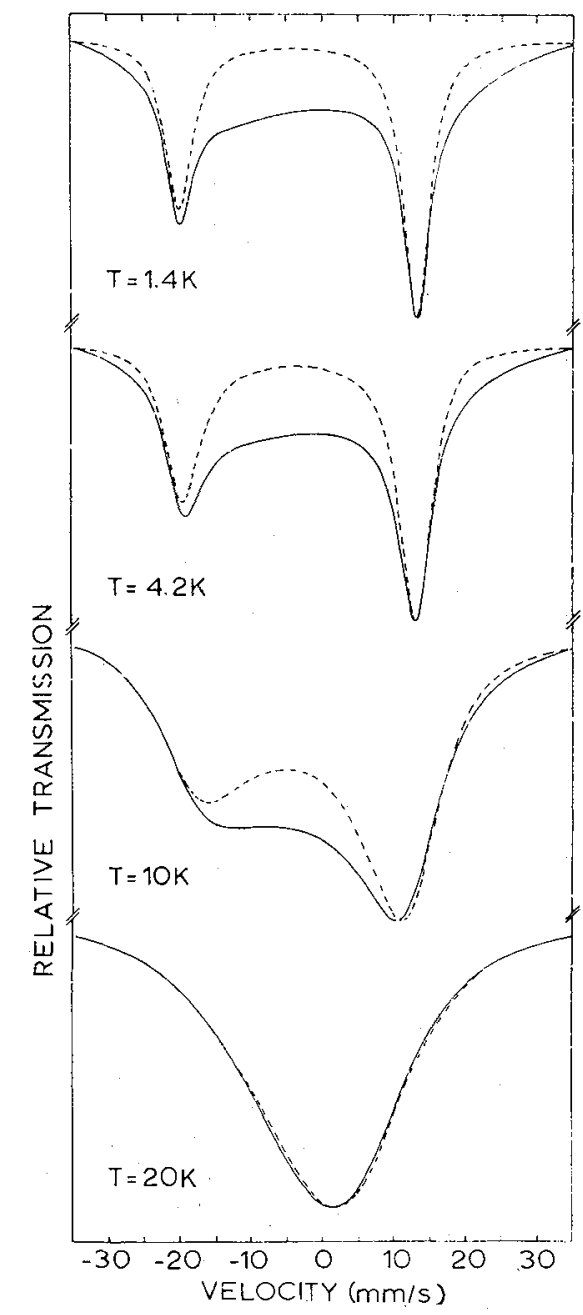

FIG. 2. - Model calculations illustrating the difference between source and absorber spectra of a $4 \mathrm{f}$ impurity due to slow intraconfigurational rearrangement. The calculations are for ${ }^{170} \mathrm{Yb}^{3+}$ with a cubic $7 \mathrm{CEF}$ ground level, and 6 and 8 levels at approximately $20 \mathrm{~K}$. Source and absorber spectra are given by solid and dashed curves. See ref. [14] for details.

trum have intensities significantly different from those corresponding to a thermal population, provided that one is working at temperatures so low as to be rable to the hyperfine splitting. A small relaxation rate not only causes incipient relaxation broadening of the resolved hyperfine lines, but also causes the total intensity of each line to shift toward its thermal value. Analysis of either the widths or the intensities may, in favorable cases, be used to determine the electronic relaxation rates. The intensity analysis does not work well unless $\omega_{\mathrm{hr}} \gtrsim k T$, whereas the width analysis may be hampered by the difficulty of determining the exact width of the single-line absorber used in a source experiment. Under optimal conditions either method can measure relaxation rates extending down to a fraction of $1 / t_{\text {nuc }}$.

An important feature of the intensity analysis is that it reflects only true relaxation processes, whereas 
the width analysis also reflects inhomogeneity broadening. In the language of magnetic resonance, the intensity analysis measures $T_{1}$ while the width analysis measures $T_{2}$. EPR measurements on Kramers $4 \mathrm{f}$ impurities yield a small residual low-temperature linewidth which cannot be accounted for by conduction-electron processes and is dependent upon the quality of the samples. The residual linewidth might plausibly be attributed to either of two mechanisms: (1) shifts in the $g$ values of the electronic levels due to random strains, and (ii) impurity-impurity interactions. Here (i) is a form of inhomogeneity broadening and (ii) is a dynamic mechanism capable of producing true relaxation flips. The EPR measurements, which are $T_{2}$ measurements, are not capable of distinguishing between the two. Accurate analyses of intensities as well as widths of the Mössbauer source spectrum of such an impurity could determine the relative importance of the two mechanisms.

5. Some applications of symmetry theory. - The theory of Mössbauer spectra with relaxation effects involves both nuclear and electronic degrees of freedom; and the electronic degrees of freedom may span several CEF levels of a $4 \mathrm{f}$ impurity (or analogous fine-structure levels of a $3 \mathrm{~d}$ impurity). Theoretical computations for realistic models can get very messy indeed if one tries to proceed by brute-force methods. We have found that a systematic exploitation of symmetry theory permits one to formulate the relaxation problem in a simpler and more economical way. In the space available, it will be impossible to give a selfcontained discussion of these methods, but we shall attempt to illustrate briefly some of the main results. An article explaining the symmetry methods in detail is slowly nearing completion and will, we hope, appear in the not-too-distant future [27].

5.1 SYMmetry ANALYSIS OF THE k-f COUPLING. The coupling between impurity and conduction electrons may be written in the form

$$
H_{\mathrm{I}}=\sum_{q}\left(K^{q}\right)^{+} F^{q}
$$

where $K^{q}$ is an operator of order of magnitude unity acting in the impurity and $F^{q}$ is an operator acting on the conduction electrons, which also incorporates the coupling strength. The spectral density is related to certain correlation functions of the $F^{q}$ operators, and the relaxation transition rates are obtained by multiplying the spectral density at the appropriate frequency by certain matrix elements of the $K^{q}$ operators. Although an arbitrary choice of the $q$ indices is possible, the theory can be simplified by choosing operators which can be labeled by symmetry indices of an appropriate symmetry group. That is, the $K^{q}$ and $F^{q}$ operators are chosen as irreducible tensor operators. This enables one to classify the various parts of the $\mathrm{k}-\mathrm{f}$ coupling in a physically meaningful way, to project the coupling or the corresponding spectral density as the finer intraconfigurational splittings are successively introduced, and to compute the corresponding relaxation effects more simply.

For example, suppose that the conduction electrons may be approximated sufficiently well as free electrons, and assume a $\mathrm{k}-\mathrm{f}$ coupling of the exchange form

$$
H_{1}=-2 J_{\mathrm{kf}} \mathbf{S . s}
$$

where $\mathbf{S}$ is the total true spin of the impurity and $\mathbf{s}$ is the density of conduction-electron spin at the origin. This coupling consists entirely of the spherical double tensor type $(\Lambda, \Sigma)=(0,1)$, meaning that the operator $\mathbf{S}$ is invariant under rotations in orbital space but is a rank-1 tensor (a pseudovector) in spin space. If we now introduce successively the LSJ coupling and CEF splitting, it is appropriate to re-express the coupling in terms of the point-group symmetry. For example, if the CEF has cubic symmetry then the coupling of exchange form, eq. (4), is found to be entirely of tensor type $\Gamma_{4}$. This symmetry classification leads to certain selection rules : for example, a coupling of type $\Gamma_{4}$ cannot induce transitions between CEF levels of types $\Gamma_{6}$ and $\Gamma_{7}$. If we had a Mössbauer source with a $\Gamma_{7}$ CEF ground level and a $\Gamma_{6}$ as the first excited CEF level, then a k-f coupling of the form (4) would yield anomalously slow depopulation of the $\Gamma_{6}$ level following the $\beta$-decay, which would show up dramatically in the Mössbauer source spectrum [14].

The true first-order Coulomb coupling between impurity and conduction electrons [29] (which includes both direct and exchange parts) contains contributions of many different spherical tensor types, although the part represented by eq. (4) can indeed be expected to be the largest. Furthermore, a coupling of SchriefferWolff type [30], when generalized to include the orbital degrees of freedom and expressed in irreducibletensor form $[31,32]$, is found to contain all spherical double tensor types up to $(\Lambda, \Sigma)=(6,1)$ with comparable coupling strengths. These terms would tend to remove the anomalously slow rearrangement of the $\Gamma_{6}$ CEF level in the above example.

The question of the relative importance of the various coupling types has received much attention in the past. Because of these intraconfigurational rearrangement effects, Mössbauer source spectroscopy is a particularly sensitive method for investigating this question.

\subsection{SIMPLIFICATION OF THE RELAXATION EQUATIONS. -} The exploitation of symmetry enables one to convert the relaxation equations into a more compact form from which symmetry-related redundancies have been eliminated. This reduction in the dimensionality of the equations which have actually to be solved is of great practical importance. Consider the example of ${ }^{170} \mathrm{Yb}^{3+}$. There are $(2 J+1)=8$ electronic states, $\left(2 I_{\mathrm{e}}+1\right)=5$ nuclear spin states in the Mössbauer excited state, and $\left(2 I_{g}+1\right)=1$ nuclear spin states in the ground state. The total dimensionality of the matrix 
equations which have to be solved according to a formulation not exploiting symmetry [3] is

$$
8 \times 5 \times 8 \times 1=320 \text {. }
$$

By elementary inspection this can be reduced to 80 , but further simplification is more difficult. Operations with an $80 \times 80$ matrix present a major computational problem even with the best modern computers.

Assuming that we have cubic point symmetry, it is found that $J=7 / 2$ reduces to the three CEF levels $\Gamma_{6}, \Gamma_{7}$ and $\Gamma_{8}$. In a symmetry-based formulation, the relaxation problems corresponding to each of these levels are found to have dimensionalities 3,3 and 10 respectively. The total dimensionality is 16 , which is duck soup for any computer worthy of the name.

5.3 SYMMETRY CLASSIFICATION OF ELECTRONIC RELAXATION. - It is interesting to consider the following problem : Given a certain manifold of electronic states, such as the CEF ground level of a $4 \mathrm{f}$ impurity, how many different real numbers are needed to specify the relaxation behaviour within this manifold at a given temperature, and how many of these rates can be determined by a given type of resonance measurement ? It turns out that an answer can be given in terms of the symmetry label of the CEF level, and the tensor type of the operator whose relaxation is being observed. In the case of EPR, one studies the motion of a magnetic moment, which is a magnetic dipole $(M 1)$ operator. In the Mössbauer effect, we are observing both the magnetic dipole and electric quadrupole $(E 2)$ electronic operators, since the Mössbauer spectrum is influenced by both $M 1$ and $E 2$ hyperfine couplings to the $4 \mathrm{f}$ shell. (We assume that the nuclear spin of the ground or excited state is large enough to support both $M 1$ and $E 2$ couplings.)

To see how things work out concretely, we list in Table II the number of real parameters needed to specify the relaxation of $M 1$ and $E 2$ electronic operators within a CEF level having any of the 8 possible symmetry types arising in the cubic group. This table can be applied provided that the relevant relaxation is all occurring within a single CEF level, and provided that any Zeeman or hyperfine splitting within this level

\section{TABLE II}

Number of real parameters specifying the magnetic relaxation of magnetic dipolar (M1) and electric quadrupolar (E2) types within cubic CEF levels of various symmetry tapes.

\begin{tabular}{|c|c|c|c|c|}
\hline $\begin{array}{l}\text { Rep. } \\
\text { label }\end{array}$ & Degeneracy & $M 1$ & $E 2$ & Total \\
\hline$\overline{\Gamma_{1}, \Gamma_{2}}$ & $\overline{1}$ & $\overline{0}$ & $\overline{0}$ & 0 \\
\hline$\Gamma_{3}$ & 2 & 0 & 1 & 1 \\
\hline$\Gamma_{4}, \Gamma_{5}$ & 3 & 1 & 2 & 3 \\
\hline$\Gamma_{6}, \Gamma_{7}$ & 2 & 1 & 0 & 1 \\
\hline$\Gamma_{8}$ & 4 & 4 & 2 & 6 \\
\hline
\end{tabular}

is small compared to $k T$ and also compared to the energy separation of neighbouring CEF levels. Then the computed spectrum depends upon the corresponding number of relaxation parameters, and under favorable conditions we might hope to fit all the parameters from the experimental data. For Mössbauer spectra, the most favorable regime for such fits is where the relaxation produces appreciable broadening without destroying the splitting pattern characteristic of the slow-relaxation limit. Each fitted relaxation parameter may be regarded as providing a piece of information about the $k-f$ coupling, and the more parameters which can be fitted, the better the k-f coupling can be characterized. However, the tensor type of the relaxation parameters is not related to that of the $\mathrm{k}-\mathrm{f}$ coupling. On the contrary, any tensor part of the k-f coupling having matrix elements within the CEF level in question will induce relaxation in all types of moment operator defined within this level.

The extra relaxation information which can, in principle, be obtained from Mössbauer spectra as opposed to EFR is that from the $E 2$ relaxation. It can be shown that $E 2$ moments do not exist within Kramers doublets [34]. The $\Gamma_{8}$ quartet of Table II, plus the corresponding quartet in the non-magnetic tetrahedral group, is therefore the only case in which $E 2$ relaxation can be measured in a Kramers level. However, E2 moments and relaxation rates are defined within several non-Kramers cubic levels as listed in Table II, and in most of the non-Kramers doublets of lower symmetry groups.

As a concrete example, let us consider the Mössbauer spectra of ${ }^{170} \mathrm{Yb}-\mathrm{Au}$ shown in figure 3. This impurity has a cubic $\Gamma_{7} \mathrm{CEF}$ ground level, with the other CEF levels at the relatively high energy of about $80 \mathrm{~K}$. The spectra in the intermediate temperature range may be discussed in first approximation assuming that neither the hyperfine-population effects discussed in $\S 4$, nor processes involving higher CEF levels, contribute. The $\Gamma_{7}$ level does not support an $E 2$ operator, and according to Table II the relaxation information at any given temperature is specified by a single real number. Features such as the relative widths or the relative intensities of the two resolved peaks do not yield any relaxation information since they are fixed by symmetry requirements. From a different point of view, these features do of course give us some very valuable information ; they are a consistency check which gives us confidence in the correctness of our analysis. In particular, they confirm that the ${ }^{170} \mathrm{Yb}$ impurity source does indeed occupy a site of undisturbed cubic symmetry.

As a second example, we consider the Mössbauer spectra of ${ }^{166} \mathrm{Er}-\mathrm{Zr}$ as shown in figure 4 . The $\mathrm{CEF}$ ground level can be identified as a Kramers doublet of symmetry type $E_{1 / 2}$, and is evidently well isolated from the nearest higher CEF levels. Within the ground level there is no $E 2$ relaxation and the $M 1$ relaxation is specified by two real parameters. The relaxed spectra 


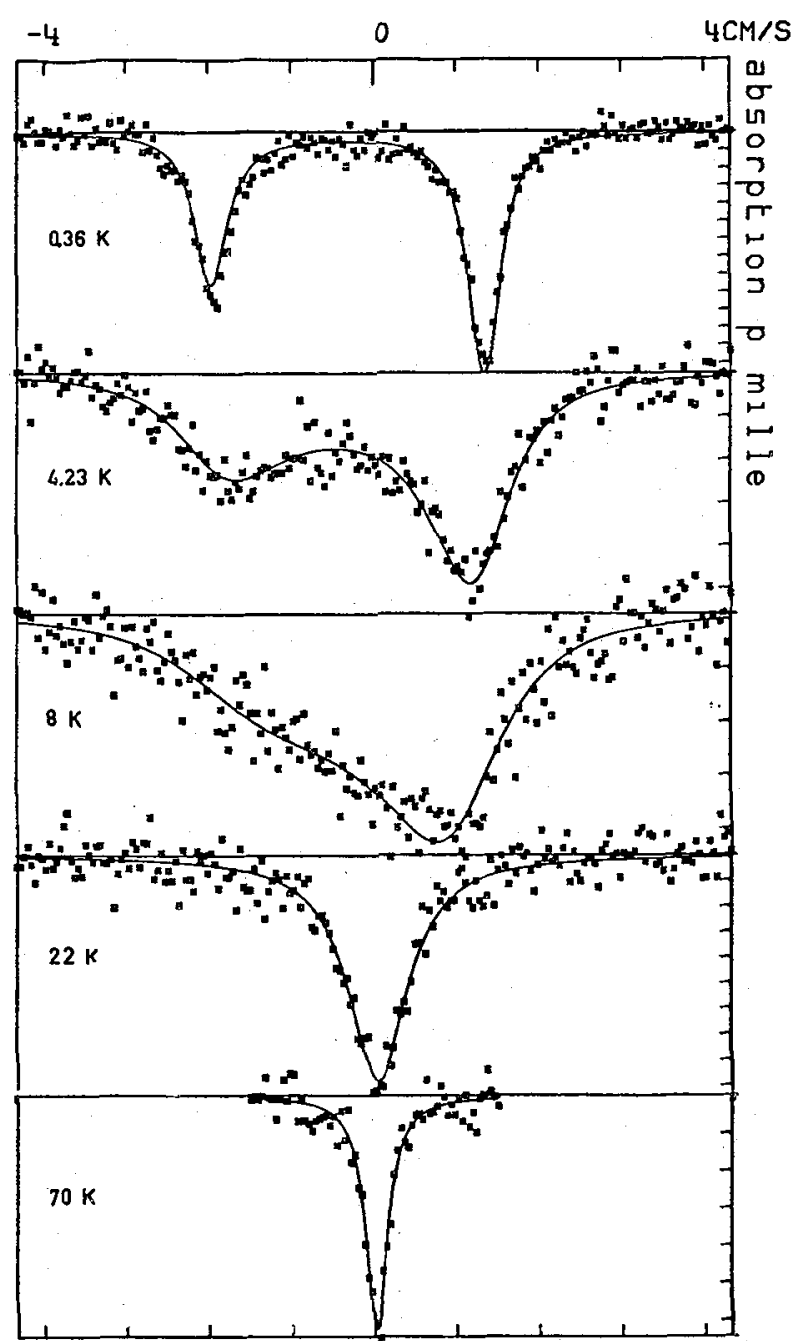

FIG. 3. - Mössbauer source spectra for ${ }^{170} \mathrm{Yb}^{3+}$ : Au. (Data provided by F. GONZALEZ-JIMENEZ and P. IMBERT ; compare ref. $[5,6]$.)

generated by the two-parameter theory are quite restricted as compared to the most general 5-line spectra one can think of. The form of the experimentally-measured spectra is rather distinctive; whereas one is accustomed to relaxation spectra in which the outermost lines show the strongest broadening [16], here the strongest broadening is shown by the 4th peak from the left, and to a lesser extent by the central peak. The successful theoretical fit shown by the solid curves therefore again constitutes a strong consistency check. Actually, these fits were made not with the most general two-parameter fit permitted by symmetry considerations, but with a one-parameter theory obtained by assuming a $\mathrm{k}-\mathrm{f}$ coupling of simple exchange type. At the time this seemed like an obvious thing to do, but in retrospect we would regard the use of such a coupling as a non-trivial assumption. The relaxation fits can therefore be regarded as giving us a valuable new piece of physical information $:$ it shows that in the particular case of Er- $\underline{\mathrm{r}}$ the exchange coupling is domi-

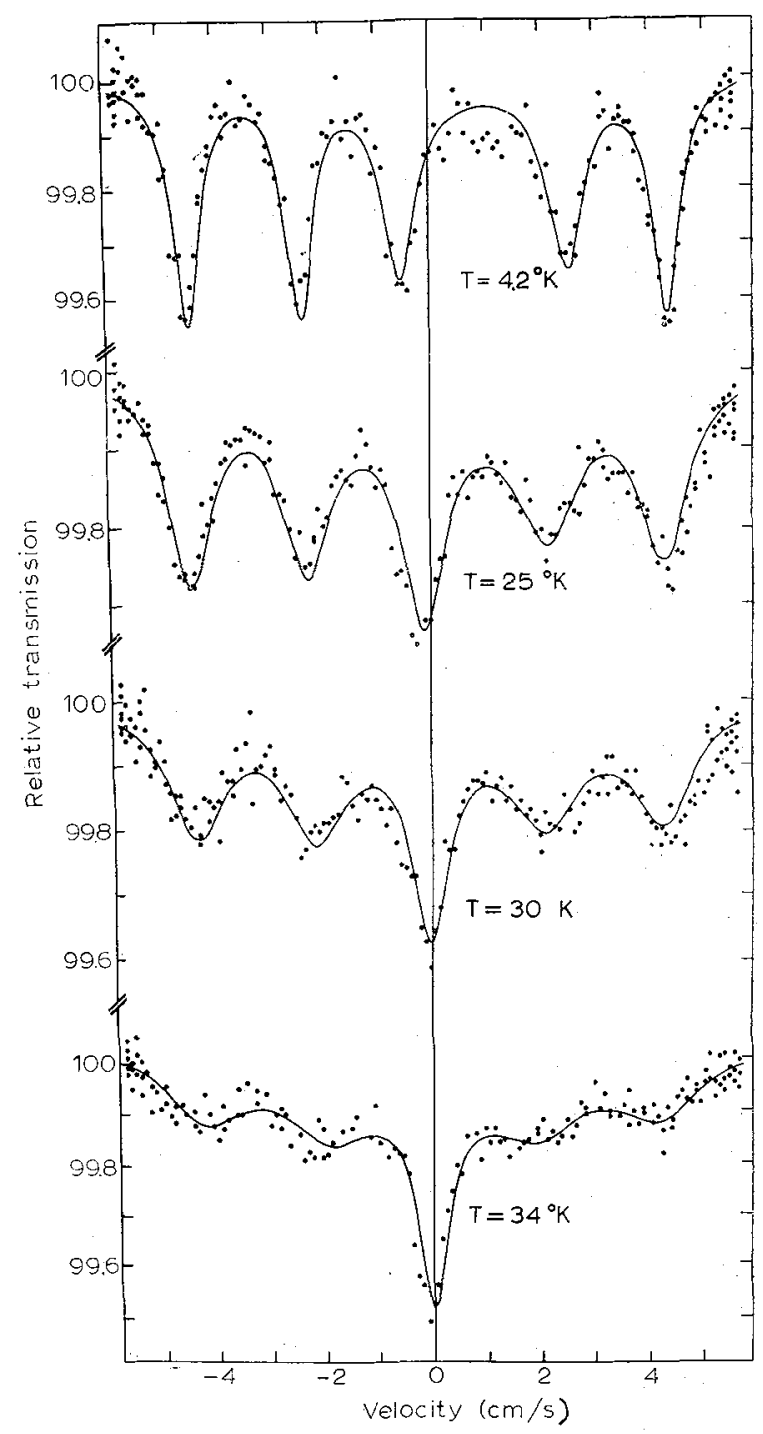

FIG. 4. - Mössbauer source spectra for $166 \mathrm{Er}: \mathrm{Zr}$, from ref. $[1,3]$.

nant compared to other contributions such as the generalized-Schrieffer-Wolff coupling.

\section{6. - Comparison between Mössbauer spectroscopy} and EPR. - EPR was the first experimental method to be used for the study of the magnetic relaxation of dilute impurities in metals, and the investigations made by this method still greatly outnumber those made by Mössbauer spectroscopy [19]. One might ask whether there is anything new to be gained by the Mössbauer method. To answer this question, it seems worthwhile to summarize in semi-tabular form the points of comparison between the two methods (some of which have already been mentioned).

6.1 EXPERIMENTAL DisadVAntages OF MösSBaUER SPECTROSCOPY. - (1) A Mössbauer isotope is not available for some impurities. 
(2) Metallurgy must often be done with radioactive impurities. Furthermore, the effects of clustering tend to show up more strongly in Mössbauer spectra, so the metallurgical requirements are more severe $[11,33]$.

6.2 EXPERIMENTAL ADVANTAges OF MÖsSBAUER SPECTROSCOPY. - (1) No external field need be used. In particular, this means that no special direction is established with respect to crystalline axes, so wellresolved resonances can be seen in polycrystalline samples even in systems of lower-than-cubic symmetry.

(2) Measurements at extreme low temperatures, in particular in a dilution refrigerator, are practical [18]

(3) A useful resonance signal can typically be followed to higher temperatures than with EPR.

6.3 ASSUMING BOTH METHODS TO BE EXPERIMENTALLY FEASIBLE, WHAT ARE THE DIFFERENCES IN THE INFORMATION WHICH CAN BE GAINED ? - (1) Analysis of the splitting pattern in the extreme slow-relaxation limit may yield somewhat different information. The comparison depends upon the specific case at hand, but one generally valid remark is that the Mössbauer spectrum is more sensitive to electric quadrupolar effects.

(2) Bottlenecking and exchange narrowing do not occur in Mössbauer spectroscopy, for the fundamental reasons mentioned in $\S 1$. A comparison of the two measurements might therefore be useful in investigating these effects. The comparison should be the most pronounced in the case of an S-state ion such as $\mathrm{Eu}^{2+}$.

(3) Within a given CEF level, the Mössbauer spectrum is influenced by relaxation rates of the types $M 1$ and $E 2$, in contrast to EPR, which is influenced only by the $M 1$ rates. The $E 2$ rates can therefore be determined by measurement of the Mössbauer spectrum, especially if EPR spectra are available for comparison. The new rates imply new information about the form of the $\mathrm{k}-\mathrm{f}$ coupling.

(4) Relaxation transitions between different CEF levels imply further information about the form of the $\mathrm{k}-\mathrm{f}$ coupling, especially information related to the presence or absence of selection rules. The relaxation between different CEF levels shows up especially well in Mössbauer source spectra due to effects associated with the intraconfigurational rearrangement [14].

This listing makes it clear that the two methods are complementary : they are sufficiently similar to provide cross-checks, but sufficiently different to provide extra information when both can be used. It is gratifying to note that checks to within experimental accuracy $[11,33]$ have been found in all cases so far where direct comparison was available. (The best possibilities for checks are provided by the magnetic hyperfine coupling constant and by the single relaxation-rate parameter defined within a $\Gamma_{6}$ or $\Gamma_{7} \mathrm{CEF}$ level in a cubic CEF.)

In view of these comparisons, we have a constructive interference between slow-relaxation Mössbauer spec- troscopy and EPR, which should become even more valuable in the future as increasingly complicated systems are investigated.

7. Rearrangement in the Kondo effect. - In earlier sections we have discussed two types of slow-rearrangement effects which may affect the Mössbauer source spectrum : that associated with intraconfigurational excited electronic states, and that associated with population of the hyperfine levels within the electronic ground level. A third type of rearrangement effect may arise when the impurity shows a Kondo effect, as we shall now briefly discuss.

The disruption of the electronic system caused by the change in nuclear charge extends to the conduction electrons, which are left in a randomized, highlydisturbed state. The charge rearrangement of the conduction electrons, i. e. their rearrangement into a distribution which gives the appropriate charge screening of the new electronic state of the impurity, is characterized by energies of $\mathrm{eV}$ magnitude and hence goes extremely fast on the Mössbauer time scale. The formation of a Kondo compensation cloud begins after this quasi-instantaneous charge rearrangement and is characterized by rates of order $k T_{\mathrm{K}}$, where $T_{\mathrm{K}}$ is the Kondo temperature. We are interested in cases where $k T_{\mathrm{K}} \lesssim \omega_{\mathrm{hf}}$, since otherwise the electronic relaxation will be too fast to permit us to study relaxation via the Mössbauer spectrum. We expect that the Kondo effects in the source spectrum, where the Kondo compensation requires time to form, should be weaker than those in the absorber, where the Kondo compensation is already present when the gamma transition begins.

This Kondo rearrangement phenomenon is very interesting from a theoretical point of view, since in principle study of the source spectra enables one to obtain some information about the time development of the Kondo effect. Furthermore, a correct calculation of the source spectrum poses an interesting theoretical problem. Although the formal relation between source and absorber spectra is quite simple in the second-order theory, this is no longer so in the case of a higher-order perturbation development. The absorber spectra should be calculated using a fully compensated impurity, formed by allowing the k-d or k-f coupling to be adiabatically switched on from $t=-\infty$. In accordance with the above discussion, the source spectrum should be calculated starting from a state in which the conduction electrons have completed their charge rearrangement only. That is, the density matrix describing the system at the start of the gamma emission $(t=0)$ should consist of a Fermi distribution for the conduction-electron states and a fully random distribution over all the relevant intraconfigurational and hyperfine levels of the impurity. The k-d or k-f coupling is regarded as acting only for $t>0$, since all its effects at $t<0$ are wiped out in the disruption caused by the nuclear transformation and can have no 
effect on the emission spectrum. This means that different fundamental perturbation expansions must be used in the source and absorber cases.

Gonzalez et al. [6-8] have reported $\log T$ dependences in the fitted electronic relaxation rates for ${ }^{170} \mathrm{Yb}-\mathrm{Au}$, which are presumably of Kondo origin. However, in our opinion their theoretical analysis is not entirely satisfactory since the Kondo rearrangement has not been taken correctly into account.

8. Future problems. - Our discussion, and most previous experimental studies, have concentrated on spectra of slowly-relaxing non-S state Kramers $4 f$ impurities. Such studies have proved most fruitful and we hope that more will be made in the future. In what follows we should like to comment briefly on further, somewhat different, problems for future investigation.

(1) Relaxation of $4 \mathrm{f}$ impurities in superconductors : Immediately subsequent to the BCS theory, experimental and theoretical work on NMR in super-conductors indicated a most interesting behaviour of the nuclear relaxation rate as the temperature is lowered through the superconducting transition temperature [35]. Very similar effects are to be expected in the relaxation of paramagnetic impurities. Although studies have been made by EPR [36], the necessity of an external magnetic field in such experiments causes serious complications due to the destruction of the superconductivity by the field, and, conversely, the Meissner screening of the field by the superconductivity. Mössbauer spectroscopy, which can be done without an external field, should eliminate these difficulties.

(2) S-state impurities : Such impurities are subject to a very weak CEF, whose magnitude is typically a fraction of a degree $K$. Such splittings have been studied by EPR, but not yet by the Mössbauer method. Since the CEF and hyperfine Hamiltonians are of comparable magnitude, they must be diagonalized simultaneously. Studies on $\mathrm{Eu}^{2+}$, especially in conjunction with EPR, would be interesting.

(3) Non-Kramers $4 f$ impurities: The spectra of such impurities in metals, according to a recent [19] consensus, have not yet been observed by EPR. Aside from the possibility that the CEF ground level may be a singlet, there are two reasons for this difficulty : (i) A non-Kramers ion, unlike a Kramers ion, may have its degeneracies lifted by low-symmetry CEF contributions associated with random strains. (ii) In all cases except the non-Kramers triplets arising in cubic or tetrahedral symmetry, the $g$-value perpendicular to the principal axis vanishes, making it more difficult to excite the EPR. This difficulty is not fatal in insulators, but it is more serious in metals, where one has intensity problems anyhow due to the skin depth. The second difficulty does not apply in Mössbauer spectroscopy, where the total signal strength is determined only by the nuclear radiation properties. This permits one to hope that slow-relaxation Mössbauer spectra might be seen for non-Kramers ions (such as $\mathrm{Tm}^{3+}$ ) in high-quality crystals. However, the failure to observe EPR even in triplet CEF levels suggests that the first difficulty (which applies to Mössbauer measurements as well) is also serious. Particularly interesting would be the observation of slow-relaxation Mössbauer spectra from the $\Gamma_{3}$ nonmagnetic doublet of the cubic group, within which EPR is intrinsically impossible.

(4) Experimental comparison of source and absorber spectra: This would be of great interest as a direct test for the intraconfigurational rearrangement effects described above. Although absorber experiments are usually impractical due to the high dilution we require, in a very light host metal such as $\mathrm{Mg}$ they may be feasible.

(5) 3d impurities : Slow-relaxation spectra of such impurities would obviously be of the greatest interest ; however, it seems necessary to seek out host materials having unusually low densities of conduction-electron states. In addition, it appears that $3 \mathrm{~d}^{6}$ is the most usual configuration of $\mathrm{Fe}$ in metals, and this, like other non-Kramers configurations, will be subject to complications from random-strain splitting [26].

9. Conclusions. - On the basis of the results summarized above, Mössbauer spectroscopy may be said to be well established as a method for the investigation of the magnetic relaxation of dilute paramagnetic impurities in metals. We would like to emphasize that this is a particularly honest method, since the large number of internal consistency checks, as well as cross-checks with EPR and other data, make the interpretation unambiguous. The physics of magnetic impurities in metals is a problem of great interest, and the Mössbauer method provides a valuable new angle of investigation. We hope therefore to see continued progress, and perhaps even solutions to some of the above-listed problems will reported at the next Mössbauer Conference !

Note added in proof. - It has been discovered that the solution for Mössbauer source spectra with rearrangement effects, derived in [14] and shown in figure 2, contains an error. A corrected version of the theory has been derived and will be published. We are indebted to F. Hartmann-Boutron, D. Spanjaard, F. GonzalezJimenez, and P. Imbert for discussions during the course of this conference which lead to discovery of the error. The discussion of the present paper, which concentrates on qualitative aspects of the rearrangement problem, does not require modification.

Although we have emphasized that the relaxation fits contain various internal-consistency checks, the method is still not completely idiot-proof. For 
example, in our work [1-3] on ${ }^{166} \mathrm{Er}-\mathrm{Zr}$ we erred as to the sign of our Doppler velocity (J. Stöhr, private communication). This somewhat alters the analysis of [2] and in particular invalidates our conclusions concerning the magnitude of the magnetic hyperfine coupling constant. Fortunately, the analysis of the relaxation given in [3] is not appreciably affected by this error.

\section{References}

[1] Hirst, L. L., Sejdel, E. R., Mössbauer, R. L., Phys. Lett. 29A (1969) 673.

[2] Hirst, L. L., Seidel, E. R., J. Phys. \& Chem. Solids 31 (1970) 857.

[3] Hirst, L. L., J. Phys. \& Chem. Solids 31 (1970) 655.

[4] Gonzalez-Jimenez, F., Imbert, P., Solid State Commun. 11 (1972) 861.

[5] Gonzalez-Jimenez, F., IMBert, P., Hartmann-BouTRON, F., Phys, Rev. B 9 (1974) 95.

[6] Gonzalez-Jimenez, F., Imbert, P., Solid State Commun. 13 (1973) 85.

[7] Hartmann-Boutron, F., Phys. Rev. B, in press.

[8] GonZalez-Jimenez, F., Hartmann-Boutron, F., Imbert, P. Phys. Rev. B, in press.

[9] Gonzalez-Jimenez, F., Cornut, B., Coqbein, B., to be published.

[10] Shenoy, G. K., StöHr, J., Kalvius, G. M., Solid State Commun. 13 (1973) 909.

[11] Stöhr, J., Shenoy, G. K., Solid State Commun. 14 (1974) 583.

[12] Stöhr, J., WAGner, W., Shenoy, G. K., Phys. Lett. 47A (1974) 177.

[13] STöHr, J., Phys. Rev. B, in press.

[14] Hirst, L. L., Stöhr, J., Shenoy, G. K., Kalvius, G. M., Phys. Rev. Lett. 33 (1974) 198.

[15] See for example : Wickmann, H. H., in Mössbauer Effect Methodology, Vol. 2, Ed. Gruverman I. J. (Plenum Press, New York) 1966 ;

SCHWEgler, H., Fortschritte der Physik 20 (1972) 251 ; Clauser, M. J., Blume, M., Phys. Rev. B 3 (1971) 583.

[16] Zinn, W., Wiedemann, W., J. Appl. Phys. 39 (1968) 839.

[17] Maletta, H., Rao, K. R. P. M., NowiK, I., Z. Phys. 249 (1972) 189.
[18] Scherg, M., Seidel, E. R., LitTerst, F. J., Gierisch, W., Kalvius, G. M., J. Physique Collq. 35 (1974) C6-527.

[19] For a recent review of EPR of dilute impurities in metals, see the proceedings of the Haute-Nendaz Conference, Archives des Sciences 27 (1974).

[20] Bleaney, B., in Proc. 3rd International Symposium on Quantum Electronics, Ed. Grivet P., Bloembergen N. (Columbia University Press) 1964, p. 595.

[21] TaO, L. J., Davidov, D., Orbach, R., Chock, E. P., Phys. Rev. B 4(1971) 5.

[22] Hirst, L. L., Z. Phys. 245 (1971) 378.

[23] Chock, E. P., Davidov, D., Orbach, R., Rettori, C., TAO, L. J., Phys. Rev. B 5 (1972) 2735.

[24] STöHR, J., private communication.

[25] Hirst, L. L., Phys. Rev, 181 (1969) 597.

[26] Hirst, L. L., Adv. Phys. 21 (1972) 759.

[27] Hirst, L. L., to be published.

[28] Abragam, A., Principles of Nuclear Magnetism (Clarendon Press, Oxford) 1961.

[29] Watson, R. E., in Hyperfine Interactions, Ed. Freeman, A. J., Frankel, R. B. (Academic Press, New York) 1967.

[30] SChriefFer, J. R., WolfF, P. A., Phys. Rev. 149 (1966) 491.

[31] Coqblin, B., Schrieffẹr, J. R., Phys. Rev. 185 (1969) 847.

[32] Hirst, L. L., Z. Phys. 244 (1971) 230.

[33] StöHR, J., Dissertation, Physik-Department der Technischen Universität München, 1974.

[34] Abragam, A., Bleaney, B., Paramagnetic Resonance of Transition-Metal Ions (Clarendon Press, Oxford) 1970.

[35] Hebel, L. C., Slichter, C. P., Phys. Rev. 113 (1959) 1504.

[36] Daridov, D., Bucher, E., Rupp, L. W., Longinottr, L. D., Phys. Rev. B 9 (1974) 2879. 